\section{Estudio comparativo del tamaño mesiodistal entre dientes homólogos en dentición permanente}

\author{
Comparative study of the mesio-distal size between homologous teeth in \\ permanent dentition
}

\begin{abstract}
Resumen
El objetivo del presente estudio fue comparar el tamaño mesiodistal entre dientes homólogos en la dentición permanente y establecer la relación del tamańo mesiodistal dental en función al género. Para este fin se midieron los tamaños mesiodistales de los dientes en los modelos de estudio tomados a 120 alumnos (60 varones y 60 mujeres) entre 12 -18 ańos de edad, de 5 Instituciones Educativas del distrito de Chaclacayo, según criterios de inclusión. Se usó un vernier centesimal con $0.02 \mathrm{~mm}$ de precisión. Los datos obtenidos fueron analizados con la prueba " $t$ " de Student. Se halló diferencia entre el diámetro mesiodistal para el género masculino en 7 dientes homólogos (maxilar: incisivos laterales, caninos, 2a premolares; mandíbula: incisivos centrales, incisivos laterales, caninos y 1amolares) y para el género femenino en 4 dientes homólogos (maxilar: incisivos centrales, caninos, 1a premolares; mandíbula: 2a premolares). Se concluyó que existe discrepancia mesiodistal significativa entre dientes homólogos en 7 dientes para el género masculino y 4 dientes para el género femenino. Se confirmó la relación entre el tamańo mesiodistal y el dimorfismo sexual, donde el género masculino presentan mayor diámetro mesiodistal que el género femenino.
\end{abstract}

Palabras clave: Dentición Permanente, Modelos dentales, Dimorfismo Sexual.

\begin{abstract}
The objective of the present study was to determine the difference mesiodistal crown between the homologous teeth in the permanent dentition and to establish the relation of the mesiodistal size, according to the gender. For this purpose, there were 120 study models that belonged to 120 students ( 60 boys and 60 girls) between $12-18$ years old of 5 Educational Institutions in Chaclacayo (Lima - Peru) according to the inclusive and exclusive criteria; the mesio-distal sizes of the teeth were measured in each model. It was used a Centesimal Vernier with $0,02 \mathrm{~mm}$ of precision. The collected data was analyzed with the " $\mathrm{t}$ " of Student for determine the significant difference. Results: There was significant the discrepancy in the mesiodistal diameter. For men in 7 homologous teeth (Maxilla: lateral incisors, canines and second bicuspids; Mandible: central incisor, lateral incisors, canines and first molars) and for Women in 4 homologous teeth (Maxilla: central incisor, canines, first bicuspids; Mandible: second bicuspid). It was concluded that there is a significative mesiodistal discrepancy between homologous teeth ( 7 teeth in men and 4 teeth in women). Also the sexual dimorphism was confirmed, the mesiodistal diameter in men were greater than women.
\end{abstract}

Keywords: Permanent Dentition, dental models, Sex Characteristics.
Artículo OriginAL

\section{Gregorio Javier, Carhuamaca León'; Luis Fernando, Pérez Vargas²; Marco Antonio Coronado Tamariz³; Hugo Javier, Luque Luque ${ }^{3}$}

1 Residente de la Segunda Especialidad de Ortodoncia de la Facultad de Odontología UNMSM.

2 Docente de la Segunda Especialidad de Ortodoncia de la Facultad de Odontología UNMSM.

3 Consulta Privada.

Correspondencia 1:

C.D. Gregorio Javier Carhuamaca León

Asociación "Villa Rica” Mz "O” Lote 34 Chaclacayo Lima-Perú

Celular: 997497868

Correo eletrónico: odonto_javier@hotmail.com/ ortodoncia.new.smile@gmail.com

Fecha de recepción: 7-2-2013

Fecha de aceptación: 23-8-2013

\section{Introducción}

El diámetro mesiodistal de los dientes es el principal causante de una buena o mala interdigitación de la oclusión, esto requiere de una medición lo más exacta posible como parte de los procedimientos del diagnóstico ortodóncico para instituir un adecuado plan de tratamiento, para lo cual es necesario el uso de un vernier digital centesimal. ${ }^{1,2,3}$

La variación de sus medidas va en aumento debido al factor multiracial en nuestro país y al dimorfismo sexual; lo que determina cambios entre uno y otro individuo; así mismo dentro del mismo sujeto existen asimetrías a nivel del plano sagital (lado derecho e izquierdo) y abarca a piezas dentales (lado derecho e izquierdo, arcada superior e inferior $)^{3}$, ${ }^{4}$. Los estudios sobre el diámetro mesiodistal, confirma la discrepancia que existe entre piezas homólogas con las arcadas dentarias, en ciertas poblaciones, en su tiempo y espacio. .,6,7,8 $^{-1}$

Para el diagnóstico y tratamiento de las maloclusiones dentarias es preciso realizar un análisis de los dientes extra- yendo una información detallada de la cara oclusal, forma, tamaño, simetría de los arcos, alineamiento dentario, giroversiones, anomalías dentarias, y la simetría homóloga entre piezas dentarias superior e inferior; ya que estas maloclusiones se debe a un aumento o disminución de piezas dentarias individuales o grupales.

Dentro del tratamiento ortodóncico uno de los métodos usados es el desgaste interproximal de dientes, para la corrección de la discrepancia óseo-dentaria, eligiendo los dientes a desgastar 
aleatoriamente en grupo y no individual; y esto se da por no considerar la discrepancia mesiodistal de los dientes homólogos, lo cual sabiendo esto llevaría al correcto desgaste interproximal de dientes. Y lo más importante tener una herramienta más para tal medición, llevando al ortodoncista a ser preciso. ${ }^{4,5,10,11}$

El propósito del estudio fue comparar el tamaño mesiodistal entre los dientes homólogos en la dentición permanente y establecer su relación con el género (dimorfismo sexual), siendo medido en los modelos de estudio ${ }^{12}$. Es importante conocer la incidencia y valorar la discrepancia en nuestra población, en un determinado tiempo y espacio.

\section{Material y métodos}

Estudio descriptivo, transversal en 120 pares de modelos de estudio, de forma probabilística y aleatoriamente de los estudiantes de secundaria entre 12 y 18 años de edad de las Instituciones Educativas Nacionales del distrito de Chaclacayo ( ${ }^{\circ} 1199$ Mariscal Ramón Castilla, con 31 alumnos: 13 varones y 18 mujeres; $N^{\circ} 1218$ San Luis María de Monfort, con 12 alumnos: 5 varones y 7 mujeres; $\mathrm{N}^{\circ} 1217$ Jorge Basadre, con 12 alumnos: 6 varones y 6 mujeres; $\mathrm{N}^{\circ}$ 1188 Juan Pablo II, con 7 alumnos: 4 varones y 3 mujeres; Felipe Santiago Estenos, con 58 alumnos: 32 varones y 26 mujeres) que cursaban el ańo 2006. De un total de 1134 estudiantes que cursaban el año 2006 se seleccionaron 120(60 varones y 60 mujeres) escogidos proporcionalmentea a la población escolar de cada institución que cumplían con los siguientes criterios de inclusión: Dentición permanente desde el incisivo central hasta la primera molar, descendientes de padres y abuelos peruanos, no padecer de enfermedades sistémicas, sin tratamiento ortodóncico, sin hábitos nocivos que implique desgaste (bruxismo, morder objetos); con normooclusión, hasta un apiñamiento dentario leve; dientes sin faceta de desgaste dentario (oclusal e interproximal), sin fracturas coronarias, caries, ni restauraciones en oclusal e interproximal; sin algún tipo de anomalía dentaria de forma, tamaño y número; con buena o regular higiene oral y ausencia de sarro; sin retención dentaria que impida el copiado de la impresión de los dientes y/o espacios debajo del punto de contacto por recesión gingival interproximal.

Con la debida autorización de los padres u apoderados, se realizó el examen clínico dental. Previo cepillado dental, se procedió al registro de la oclusión con cera Kavex, llevando a relación céntrica y mordiendo hasta su máxima intercuspidación, con este registro se seleccionó la cubeta a la medida y se procedió a la toma de impresión, con Alginato "Jeltrate", haciendo el vaciado inmediatamente con yeso ortodontico "ortoguix" (para evitar cambios dimensionales), luego de la desinfección y limpieza de las superficies de la impresión. El registro de oclusión y los modelos de estudio se guardaron codificados juntamente con su ficha clínica. Se realizó el zocalado y recorte de cada modelo de estudio, sin el acabado final del modelo (para evitar la deformación en la anatomía dentaria). ${ }^{3}$

Se tomó cada modelo al azar, para medir el tamaño mesiodistal de cada pieza dentaria superior e inferior, Austros determinó de que no existe diferencia significativa entre medir en boca y medir en modelos ${ }^{16}$, la medición se realizó con un vernier digital de puntas afiladas, con precisión de $0,02 \mathrm{~mm}$ (modelo MT-00855 Uyustools Profesional), debido a la precisión que brinda el calibre digital según los autores ${ }^{1,2,3}$.

El asistente hizo las anotaciones de las medidas respectivamente en la ficha

\section{Tabla 1. Promedio del Tamańo Mesiodistal y Discrepancia Mesiodistal entre dientes homólogos en Varones.}

$\left(^{*}\right)$ Valores significativos en el análisis de t de Student

\begin{tabular}{|c|c|c|c|c|c|c|c|c|}
\hline Arcada & Pieza & Derecho & $\begin{array}{c}\text { Desviación } \\
\text { Estándar }\end{array}$ & Izquierdo & $\begin{array}{l}\text { Desviación } \\
\text { Estándar }\end{array}$ & Discrepancia & $\begin{array}{c}\text { tde } \\
\text { Student }\end{array}$ & $\begin{array}{c}\text { Significancia } \\
\text { Bilateral }\end{array}$ \\
\hline & IC & 8.95 & 0.48 & 8.96 & 0.52 & 0.01 & -0.475 & 0.637 \\
\hline $\begin{array}{l}\text { S } \\
\text { U }\end{array}$ & IL & 7.48 & 0.47 & 7.43 & 0.48 & 0.05 & 1.482 & 0.144 \\
\hline $\begin{array}{l}P \\
F\end{array}$ & C & 8.54 & 0.45 & 8.49 & 0.41 & 0.04 & 1.618 & 0.111 \\
\hline $\begin{array}{l}R \\
\text { I }\end{array}$ & $\begin{array}{l}1^{\mathrm{a}} \\
\mathrm{PM}\end{array}$ & 7.68 & 0.32 & 7.69 & 0.38 & 0.01 & -0.519 & 0.606 \\
\hline $\begin{array}{l}0 \\
R\end{array}$ & $\begin{array}{l}2^{\mathrm{a}} \\
\mathrm{PM}\end{array}$ & 7.39 & 0.33 & 7.45 & 0.37 & 0.06 & -1.810 & 0.075 \\
\hline & $1^{\mathrm{a}} \mathrm{M}$ & 11.20 & 0.49 & 11.21 & 0.50 & 0.01 & -0.419 & 0.677 \\
\hline & IC & 5.68 & 0.28 & 5.66 & 0.33 & 0.02 & -1.119 & 0.268 \\
\hline $\begin{array}{l}\mathrm{I} \\
\mathrm{N}\end{array}$ & IL & 6.32 & 0.33 & 6.36 & 0.35 & 0.03 & 1.492 & 0.141 \\
\hline $\begin{array}{l}F \\
E\end{array}$ & C & 7.39 & 0.39 & 7.44 & 0.39 & 0.05 & 2.087 & $0.041^{*}$ \\
\hline $\begin{array}{c}R \\
\text { l }\end{array}$ & $\begin{array}{c}1^{\mathrm{a}} \\
\mathrm{PM}\end{array}$ & 7.41 & 0.36 & 7.41 & 0.39 & 0.00 & 0.076 & 0.940 \\
\hline $\begin{array}{l}0 \\
R\end{array}$ & $\begin{array}{l}2^{\mathrm{a}} \\
\mathrm{PM}\end{array}$ & 7.68 & 0.40 & 7.70 & 0.40 & 0.02 & 0.533 & 0.596 \\
\hline & $1^{\mathrm{a}} \mathrm{M}$ & 12.03 & 0.49 & 12.07 & 0.52 & 0.04 & 1.781 & 0.080 \\
\hline
\end{tabular}

de recolección de datos; este procedimiento se realizó tres veces en diferentes ocasiones, sin saber de qué modelo se trataba. En cada ocasión el modelo de estudio se midió dos veces; cuando la diferencia entre ambas mediciones fue menor a $0.2 \mathrm{~mm}$, la medida mayor fue registrada en la ficha, y cuando la segunda medida difirió en más de 0,2 $\mathrm{mm}$, una tercera medida se llevó a cabo y se anotó en la ficha ${ }^{5}$. Las mediciones fueron tomadas a luz del día e intercalando de modelo en modelo un periodo de 10 minutos, evitando la fatiga del investigador (fatiga visual y manual). ${ }^{13}$

Se utilizó el programa SPSS versión 15. Se halló el promedio, desviación estándar y se usó la prueba "t" de Student para muestras dependientes y para muestras independientes con un $95 \%$ de nivel de confianza.

\section{Resultados}

Para determinar la discrepancia mesiodistal entre dientes homólogos en varones y en mujeres (Ver Tabla 1 y 2) se realizó el análisis de significancia a partir de los promedios del tamaño mesiodistal, encontrándose que la discrepancia no es significativa 
Tabla 2. Promedio del Tamaño Mesiodistal y Discrepancia Mesiodistal entre dientes homólogos en mujeres.

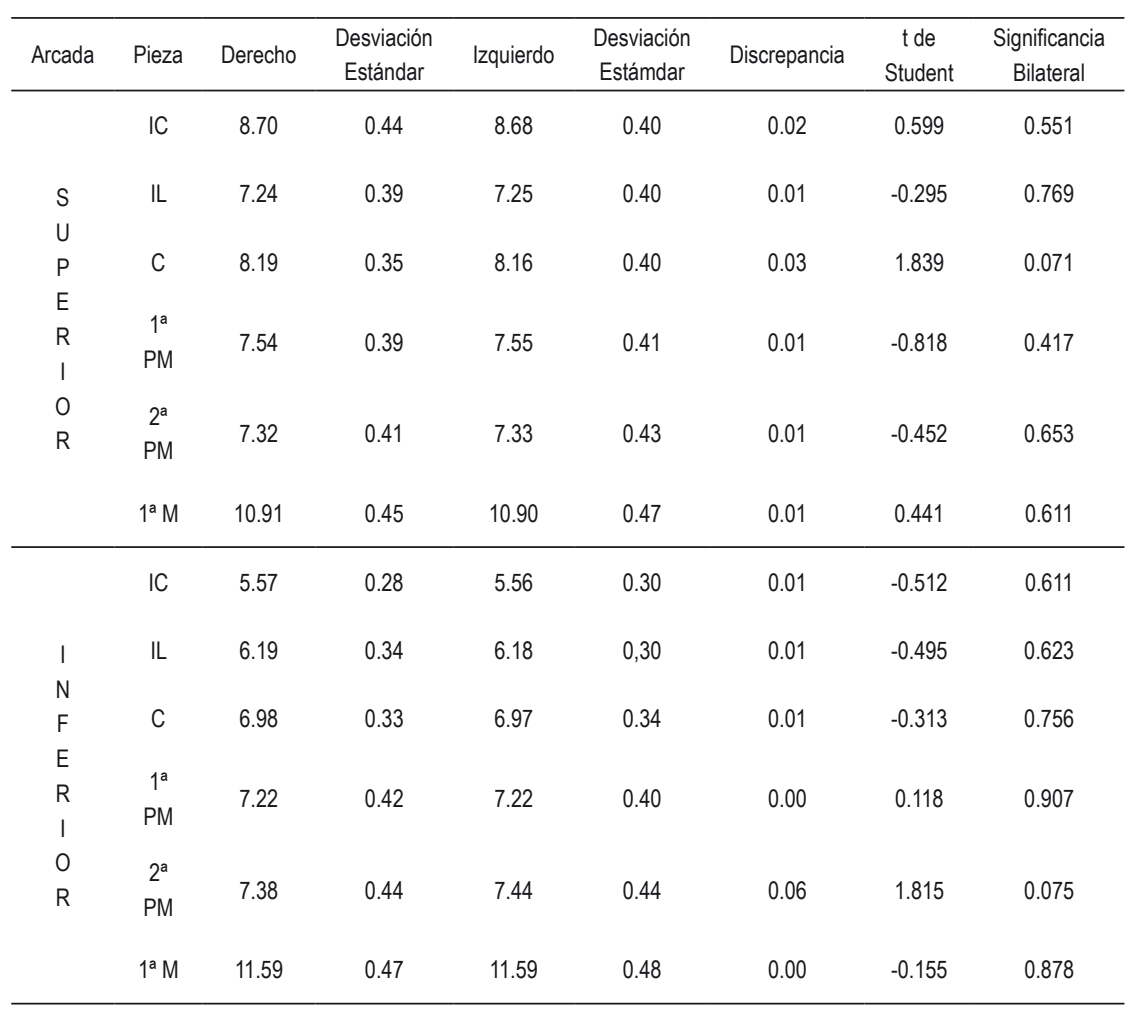

Comparando los tamaños mesiodistales entre varones y mujeres, resultó que hay diferencia estadística significativa entre todas las piezas dentarias, excepto entre la $2^{\text {da }}$ PM superior, determinando que la dimensión mesiodistal dentaria de los varones es mayor que de las mujeres. (Ver Tabla 3).

Tabla 3. Comparación de los tamaños mesiodistales entre varones y mujeres. $\left(^{*}\right)$ Valores significativos en el análisis de t de Student

\begin{tabular}{|c|c|c|c|c|c|c|c|c|}
\hline Arcada & $\begin{array}{c}\text { Pieza } \\
\text { Dentaria }\end{array}$ & Masculino & $\begin{array}{c}\text { Desviación } \\
\text { Estándar }\end{array}$ & Femenino & $\begin{array}{c}\text { Desviación } \\
\text { Estándar }\end{array}$ & Diferencia & $\begin{array}{c}\mathrm{t} \text { de } \\
\text { Student }\end{array}$ & $\begin{array}{c}\text { Significancia } \\
\text { Bilateral }\end{array}$ \\
\hline \multirow{6}{*}{$\begin{array}{l}S \\
U \\
P \\
E \\
R \\
I \\
O \\
R\end{array}$} & IC & 8.9541 & 0.49415 & 8.6916 & 0.40249 & 0.26250 & 3.220 & $0.002^{*}$ \\
\hline & $\mathrm{IL}$ & 7.4544 & 0.45303 & 7.2437 & 0.37616 & 0.21075 & 2.889 & $0.005^{*}$ \\
\hline & C & 8.5145 & 0.41770 & 8.1741 & 0.36733 & 0.34042 & 4.577 & $0.000^{*}$ \\
\hline & $1^{\text {a } P M}$ & 7.6853 & 0.32480 & 7.5455 & 0.39550 & 0.13983 & 2.006 & $0.049^{*}$ \\
\hline & $2^{\mathrm{a}} \mathrm{PM}$ & 7.4205 & 0.32642 & 7.3224 & 0.40652 & 0.09808 & 1.318 & 0.193 \\
\hline & $1^{\mathrm{a}} \mathrm{M}$ & 11.2030 & 0.48592 & 10.9074 & 0.44966 & 0.29558 & 3.428 & $0.001^{*}$ \\
\hline \multirow{6}{*}{$\begin{array}{l}\text { I } \\
N \\
F \\
E \\
R \\
\text { I } \\
0 \\
R\end{array}$} & IC & 5.6676 & 0.29785 & 5.5633 & 0.27810 & 0.10425 & 2.133 & $0.037^{*}$ \\
\hline & $\mathrm{IL}$ & 6.3375 & 0.32987 & 6.1808 & 0.30752 & 0.15667 & 2.870 & $0.006^{*}$ \\
\hline & C & 7.4154 & 0.37613 & 6.9778 & 0.32541 & 0.43758 & 6.320 & $0.000^{*}$ \\
\hline & $1^{\text {a } P M}$ & 7.4074 & 0.33495 & 7.2178 & 0.39274 & 0.18967 & 2.691 & $0.009^{*}$ \\
\hline & $2^{\mathrm{a}} \mathrm{PM}$ & 7.6904 & 0.38109 & 7.4104 & 0.42228 & 0.28000 & 3.592 & $0.001^{*}$ \\
\hline & $1^{\mathrm{a}} \mathrm{M}$ & 12.0516 & 0.49408 & 11.5919 & 0.46538 & 0.45967 & 5.675 & $0.000^{*}$ \\
\hline
\end{tabular}

\section{Discusión}

El diámetro mesiodistal de las piezas dentarias permanentes en los hombres y en las mujeres concuerda en $30 \%$ con los valores obtenidos por Pineda M. y Liviac T. ${ }^{4}$

En este estudio se halló que existe diferencia numérica del tamaño mesiodistal entre dientes homólogos excepto entre las primeras premolares inferiores en varones $y$ primeras premolares y molares inferiores en mujeres, pero no estadísticamente significante.

Este resultado no coincide con Bishara y Col. ${ }^{8}$ que en el año 1986 en una población de México y EEUU, encuentran $^{9}$ piezas dentarias con discrepancia significativa en el diámetro mesiodistal de dientes homólogos, sin mencionar que piezas dentarias. Así mismo no coincide con Austro Martínez y Col.4 que en el año 2003 en una población de Andalucía Oriental, existe diferencia significativa en el diámetro mesiodistal de dientes homólogos de la $2^{\text {a }}$ premolar superior en varones, y del canino superior en mujeres.

Se coincide con los resultados de los trabajos de Hattab y Col. ${ }^{9}$ en el año 1996 en una población de Jordanos, Otuyemi y Noar. ${ }^{10}$ en el año 1996 en una población Nigeriana y Británica, Paredes Gallardo y Col. ${ }^{11}$ en el año 2003 en una población de Valencia que estudiaron según su bibliografía a los incisivos laterales superiores y segundas premolares inferiores, y Sahim Saglam, Aynur Medine y Col. ${ }^{12}$ en el año 2004 estudiaron en sujetos con fluorosis y sin fluorosis de Anakara-Turquía encontrando que no existía discrepancia significativa para ambas muestras; todos ellos no encontraron discrepancias significativas en la comparación del diámetro mesiodistal de todas las piezas dentarias con su homólogo.

También se observó la diferencia entre el género (dimorfismo sexual) para el tamańo mesiodistal de las piezas dentarias donde el tamaño de todos los dientes permanentes en los varones es más grande que el de las mujeres, pero no estadísticamente significativo. Coincidiendo y discrepando con algunos los estudios de: Pineda y Liviac. ${ }^{7}$ en el ańo 1975 en Lima, Hattab y Col. ${ }^{9}$ en el año 1996 en Jordanos, Santoro, Margherita y Col. ${ }^{13}$ en el ańo 2000 en una población de América Dominicana con raza mixta, Díaz y Fariñas. ${ }^{14}$ en el año 2000 en una población de Cuba, Adeyemi e Isiekwe. ${ }^{15}$ en el año 2003 que determinó que los dientes del sexo masculino 
son más grandes que el sexo femenino siendo $3.31 \mathrm{~mm}$. y $1.37 \mathrm{~mm}$. mayores la suma total de los dientes maxilares y mandibulares respectivamente en los varones que en las mujeres, Ruiz Bravo.5 en el año 2004 en la ciudad de Talca-Chile, Paulino y Col. ${ }^{16}$ en el año 2005 en una población espańola, Haralabakis y Col. ${ }^{17}$ en el año 2006 en una población de Atenas-Grecia, Singh y Goyal. ${ }^{18}$ en el año 2006 en niños del Norte de India; mientras que para Bishara y Col. ${ }^{8}$ en el año 1986 en una población de México y EEUU encontró que sólo los caninos y molares son significativamente más grandes en los hombre que en la mujeres en su diámetro mesiodistal, y Ling y Wong ${ }^{19}$ en el ańo 2007 encontraron que todos los dientes son significativamente más grandes en los hombre que en la mujeres en su diámetro mesiodistal, con excepción del incisivo central inferior.

Debido a que existe diferencias en tamaño mesiodistal entre dientes homólogos, no siendo significantes estadísticamente, pero de posible significancia clínicamente a la observación del ortodoncista, lo que se deduce que podrían causar una desarmonía oclusal por incremento de tejido dentario, también estarían causando una maloclusión dentaria. Se ha determinado que las piezas dentarias que mayor diferencia presentan son las premolares e incisivos laterales y caninos tanto en el hombre como en la mujer ya sea en diferente orden, causarían una discrepancia Bolton, lo que llevaría consigo un no adecuado engranaje oclusal, también se estaría aludiendo este exceso de tejido dentario para una incorrecta llave molar, canina e incisiva; por ejemplo: en algunos pacientes que presentan maloclusión clase II derecha o izquierda, puede deberse a aun aumento del tejido dentario en la arcada inferior a consecuencia de una diferencia del tamaño mesiodistal de una o varias piezas dentarias; en el caso de una maloclusión clase III derecha o izquierda, puede deberse a aun aumento del tejido dentario en la arcada superior; del mismo modo puede causar este desarreglo en las clases caninas, pronunciamiento del over jet o mordida bis a bis; otra característica importante para la estética dental es la coincidencia de la línea media dental y/o facial, que también estaría influenciada por la diferencia del tamaño mesiodistal entre los dientes homólogos

\section{Conclusiones}

No existe diferencia del tamaño mesiodistal estadísticamente significativa tanto en varones como en mujeres, pero si existe una diferencia numérica. Así mismo no hay diferencia estadísticamente significativa entre sexo masculino $y$ femenino (dimorfismo sexual), pero si ocurre que los hombres presentan mayor diámetro mesiodistal que las mujeres en todos los dientes.

\section{Referencias Bibliográficas}

1. Guinness NJ, Stephens CD. Storage of orthodontic study models in hospital units in the U.K. Journal Of Orthodontics 1992;19(3):227-32.

2. Millan G. Procedimientos de Mecanizado. Madrid: Editorial Paraninfo; 2006: 23-34

3. Moorrees CFA, Reed RB. Correlations among crown diameters of human teeth. Archives of Oral Biology. 1964;9(6):685-97.

4. Pineda MM, Liviac TR. Estudio de los diámetros mesiodistales de las coronas de la dentición permanente en una población escolar de Lima Metropolitana. Año 1976. [Tesis de Bachiller en Odontología]. Universidad Nacional Mayor de San Marcos. 1976

5. Ruiz I. Análisis de Ancho Mesio Distal Coronario y Discrepancias Dento Dentarias en Pacientes Ortodóncicos de la Ciudad de Talca. Chile. [Tesis de Titulación]. Universidad de Talca. 2004. Disponible en: http://dspace.utalca.cl/ handle/1950/1060.

6. Bishara S, Fernandez A, Jakobsen J, Fahl. Mesiodistal crown dimensions in México and The United States. The Angle Orthodontist. 1986;56(4):315-23.

Disponible en: http://www.angle.org/anglonline/?request=getabstract\&issn $=0003-3219 \&$ volum $\mathrm{e}=056 \&$ issue $=04 \&$ page $=0315$

7. Hattab FN, Khateeb S, Sultan I. Mesiodistal crown diameters of permanent teeth in Jordanians. Archives of Oral Biology. 1996;4(7):641-45.
Disponible en: http://www. ncbi.nlm.nih.gov/entrez/query. fcgi? $\mathrm{CMD}=$ search $\& \mathrm{DB}=$ pubmed

8. Santoro M, Ayoub M, Pardi V, Cangialosi T. Mesiodistal Crown Dimensions and Tooth Size Discrepancy of the Permanent Dentition of Dominican Americans. The Angle Orthodontist. 2000;70(4):303-07.

9. Paulino VS, Paredes V, Gandia JL, Cibrian RM. Evolución de las características de las arcadas dentarias en dos grupos de edad. RCOE: Revista del Ilustre Consejo General de Colegios de Odontólogos y Estomatólogos de España. 2005;10(1)31-42.

Disponible en: http://wwwscielo.isciii.es/scielo.php?script $=$ sci arttext\&pid=S1138-123X2005000 $100004 \& \operatorname{lng}=e s \& n r m=i s o \& t \operatorname{lng}=e s$

10. Paredes V, Gandia JL, Cibrian RM. Método de medición del índice de Bolton mediante la digitalización de la arcada dentaria. Ortodoncia Española: Revista de Clínica e Investigación en Ortodoncia. 2003;43(2):75-84.

11. Díaz JE, Farińas MM. Comportamiento del Índice Incisivo en Ortodoncia. Correo Científico Médico de Holguín-Cuba. 2002;6(4). Disponible en: http://www.cocmed.sld.cu/no64/n64ori4.htm

12. Austro MMD, Ostos GMJ, García BC, Pérez LL. Análisis de la simetría del tamaño dentario mesiodistal de la misma muestra en dentición mixta y permanente. Estudio longitudinal. Avances en Odontoestomatología. 2004;20(6):305-13.

13. Hunter WS, Priest WR. Errors and discrepancies in measurement of tooth size. Journal of Dental Research 1960;39(2):405-14.

14. Otuyemi O, Noar J. A comparison of crown size dimensions of the permanent teeth in a Nigerian and a British population. Euro Journal of Orthodontics 1996;18(1):62328. Disponible en: http://ejo.oxfordjournals.org/cgi/content/abstract/18/1/623

15. Sahin, Murat, Saglam. A comparison of mesio-distal crown dimensions of the permanent teeth in subjects with and without fluoro- 
sis. Euro Journal of Orthodontics 2004;26(3):279-81. Disponible en: http://ejo.oxfordjournals.org/ cgi/reprint/26/3/279

16. Adeyemi TA, Isiekwe MC. Comparing permanent tooth sizes (mesio-distal) of males and females in a Nigerian population. West African Journal of Medicine 2003;22(3):219-21.

17. Haralabakis NB, Sifakakis I, Papagrigorakis M, Papadakis G. The correlation of sexual dimor- phism in tooth size and arch form. World Journal Orthodontics 2006;7(3):254-60.

18. Singh SP, Goyal A. Mesiodistal crown dimensions of the permanent dentition in North Indian children. Journal of Indian Society Pedodontics and Preventive Dentistry 2006;24(4):192-96. Disponible en: http://www.jisppd. com/article.asp?issn $=09704388$; ye ar $=2006$; volume $=24$;issue $=4$; ;page $=192$; epage $=196$; aulast $=$ Singh
19. Ling JY, Wong RW. Tooth dimensions of Southern Chinese. Homo: internationale Zeitschrift für die vergleichende Forschung am Menschen 2007;58(1):67-73. Disponible en:http://www.ncbi.nlm.nih. gov/entrez/query.fcgi? $\mathrm{cmd}=$ Retriev e\&db=pubmed\&dopt $=$ Abstract \&l ist_uids $=17239376$ 